Copyright (c) 2005 IEEE. Reprinted from

IEEE Transactions on Power Delivery, 2005; 20:191-196

This material is posted here with permission of the IEEE. Such permission of the IEEE does not in any way imply IEEE endorsement of any of the University of Adelaide's products or services. Internal or personal use of this material is permitted. However, permission to reprint/republish this material for advertising or promotional purposes or for creating new collective works for resale or redistribution must be obtained from the IEEE by writing to pubs-permissions@ieee.org.

By choosing to view this document, you agree to all provisions of the copyright laws protecting it. 


\title{
Analysis of a Ferroresonant Circuit Using Bifurcation Theory and Continuation Techniques
}

\author{
Frank Wörnle, David K. Harrison, and Chengke Zhou
}

\begin{abstract}
In this contribution, the analysis of the dynamics of a ferroresonant circuit are presented using continuation techniques and bifurcation theory. Despite its great simplicity, this circuit can assume a diverse range of steady-state regimes including fundamental and subharmonic ferroresonance, quasiperiodic oscillations, and chaos. The system dynamics are explored through the continuation of periodic solutions of the associated circuit equations. A detailed picture is drawn of various transitions between the individual periodic steady-state regimes of the circuit. Bifurcation points are computed, revealing a clearly defined succession of periodic steady-state regimes, including the Feigenbaum route to chaos through a cascade of period doublings. The analysis presented is performed using the freely available software package XPPAUT. The contribution of this paper is to provide a detailed description of how to define the circuit equations in XPPAUT and how to conduct the interactive bifurcation analysis. The proposed approach is shown to be both computationally efficient and robust, as it eliminates the need for numerically critical and long lasting transient simulations.
\end{abstract}

Index Terms-Bifurcation, continuation, ferroresonance, XPPAUT.

\section{INTRODUCTION}

I $\mathrm{N}$ power-distribution networks, ferroresonance can occur when a de-energized phase of a transformer is suddenly energized through one of the neighboring phases. The equivalent circuit diagram is that of a nonlinear series resonance circuit as shown in Fig. 1. The resistive losses of the line and winding of the transformer are modeled by the series resistor $R_{\mathrm{s}}$ while the core losses (hysteresis, eddy currents) are collected in $R_{\mathrm{p}}$; the magnetic flux is denoted by $\Phi$ and $C$ represents the capacitance between the energizing and the de-energized phase.

The phenomenon of ferroresonance has been the focus of many investigations [1]-[4]. The term "ferroresonance" collectively describes a class of nonlinear effects which are caused by magnetic saturation. Initially restricted to periodic oscillations (fundamental and subharmonic ferroresonance), the spectrum of ferroresonant phenomena has been extended to include aperiodic steady-state regimes, such as quasiperiodicity and chaos.

The commonly adopted technique of brute-force integration is limited to the investigation of asymptotically stable steady states. To explore both stable and unstable periodic orbits (SPO and UPO), additional geometric properties of the differential equations have to be considered [5]. A suitable framework is

Manuscript received June 9, 2003. Paper no. TPWRD-00278-2003.

F. Wörnle was with the School of Engineering, Science and Design, Glasgow Caledonian University, Glasgow G4 0BA, U.K. He is now with the School of Mechanical Engineering, University of Adelaide, Adelaide, Australia.

D. K. Harrison and C. Zhou are with the School of Engineering, Science and Design, Glasgow Caledonian University, Glasgow G4 0BA, U.K.

Digital Object Identifier 10.1109/TPWRD.2004.835529

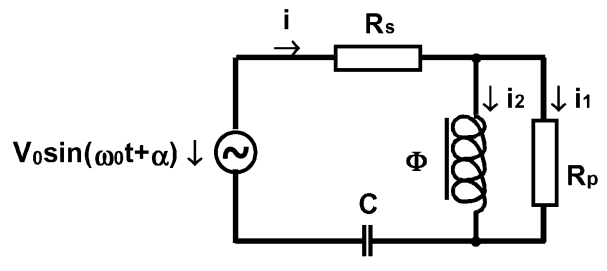

Fig. 1. Standard ferroresonant circuit highlighting the main elements.

given by bifurcation theory, a branch of applied mathematics concerned with the structural stability of ordinary differential equations under the variation of one or more parameters.

The present contribution demonstrates how results obtained from bifurcation theory can be applied to the analysis of the ferroresonant circuit shown in Fig. 1. Bifurcation diagrams are computed for the magnetic flux $(\Phi)$ as a function of the applied supply voltage amplitude $V_{0}$. The analysis is carried out using the freely available software package XPPAUT [6]. This software package combines Bard Ermentrout's phase plane explorer XPP [7] with the popular continuation package AUTO [8]. The results presented confirm the excellent suitability of this software for the numerical analysis of nonlinear dynamic systems.

\section{Continuation Techniques AND Bifurcation TheORY}

The principle of continuation is a mathematical technique in which the path of an established solution of a system of equations is followed around parameter space when a control parameter is varied [9]. The solution branch thus established can then be examined for special bifurcation points, at which a qualitative change of the preceding solution type can be observed. In dynamic systems, these qualitative changes frequently come in sequences: a stationary state is replaced by regular motion which can develop into irregular motion. The transition from regular to irregular motion is often related to the onset of chaos. Tracing the solution branches and detecting the bifurcation points, an accurate portrait can be drawn for the dynamics of the system.

All continuation techniques are based on the fundamental assumption that the solutions of an ordinary differential equation (ODE) vary continuously with the initial conditions and the parameters of the ODE. Implementing a predictor-corrector scheme, a continuation algorithm can trace the path of an already established solution as the parameters are varied. This extrapolation appears to pull the solutions along an invisible path, thereby forming the corresponding solution branch. At intersections, where a solution generates two different solution branches, the algorithm can either resume continuation of the prevailing branch or perform branch switching to the intersecting branch. 


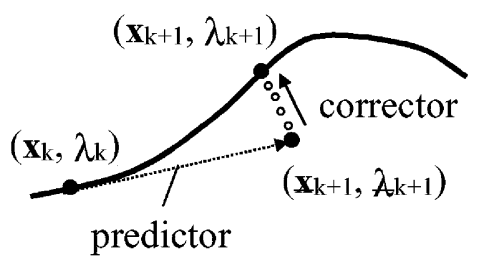

Fig. 2. Principle of arc-length continuation.

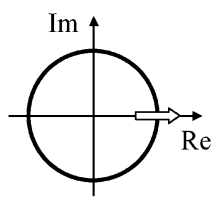

$a$

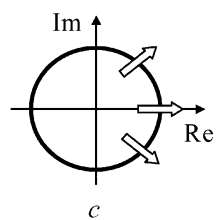

Fig. 3. Three scenarios for the loss of stability of a solution: (a) Fold bifurcation. (b) Flip bifurcation. (c) Secondary Hopf bifurcation.

Fig. 2 illustrates the principle of arc-length continuation. Denoting the system parameter by $\lambda$ and the n-dimensional state vector by $\mathbf{x}$, a particular solution can be expressed by the $n+1$-dimensional (1-D) vector $\left(\mathbf{x}_{k}, \lambda_{k}\right)$. The index $k$ denotes the $k$ th solution of the ongoing continuation process. The predictor determines a likely neighboring solution by linear extrapolation of the established solution branch in the direction of the tangent vector of the current solution. The predicted state $\left(\underline{\mathbf{x}}_{\mathrm{k}+1}, \underline{\lambda}_{k+1}\right)$ is then corrected to the true solution $\left(\mathbf{x}_{k+1}, \lambda_{k+1}\right)$ using an iterative algorithm such as the gradient descending Newton-Raphson method (small circles in Fig. 2). As this corrector is independent of the system dynamics, the continuation method is able to converge to solutions irrespective of their stability properties. The number of iterations required by the corrector can be used to adjust the step size of the predictor [10]. Loss of stability is encountered when a multiplier leaves the unit circle. This can occur in any of the following three ways (Fig. 3).

The methodology of continuation requires at least one solution of the system equations to be known. A suitably designed homotopy can be used to find this initial solution [10]. Alternatively, the dynamic equations can be integrated until the steady state has been reached. For small supply voltages (e.g., $\mathrm{V}_{0}=10 \mathrm{~V}$, the current $i$ of the resonance circuit in Fig. 1 has a periodic attractor. The continuation can thus be initiated with the set of state variables which result from a sufficiently long time-domain simulation at $\mathrm{V}_{0}=10 \mathrm{~V}$.

A useful byproduct of the continuation procedure is information about the stability properties of the established solutions. In the present case of the above ferroresonant circuit, only periodic steady-state solutions [limit cycle oscillations (LCOs)] are considered. The stability of an LCO can be assessed from the corresponding characteristic multipliers or Floquet multipliers. These multipliers represent a generalization of the eigenvalues of an equilibrium point, as they describe the local contraction or expansion rate of phase space near the limit cycle of interest. Floquet theory is based on the observation that a periodic so- lution can be represented through a fixed point of an associated Poincaré map [5], [11]. Consequently, the stability of an LCO can be determined by assessing the stability of the corresponding fixed point of this Poincaré map. Linearization of the Poincaré map at the fixed-point results in a linear discrete-time system which describes the period-to-period evolution of oscillations in the vicinity of the LCO. The Floquet multipliers are now given by the eigenvalues of the Jacobian of this linear system, the so-called monodromy matrix. Stable characteristic multipliers, being the eigenvalues of a map, are found inside the unit circle $(|\sigma|<1)$; conversely, unstable periodic limit cycles have at least one characteristic multiplier outside the unit circle.

A fold bifurcation is encountered when a single real eigenvalue of the monodromy matrix crosses the unit circle at +1 [Fig. 3(a)]. This bifurcation corresponds to the widely studied jump phenomenon of a ferroresonant circuit [i.e., the sudden increase (decrease) of the current amplitude when the supply voltage exceeds (falls below) a particular threshold $\left.\mathrm{V}_{\text {on }}\left(\mathrm{V}_{\mathrm{off}}\right)\right]$. The second scenario in which the stability of a solution can be lost is the flip bifurcation or period-doubling bifurcation. This case is given when a single real eigenvalue crosses the unit circle at -1 [Fig. 3(b)]. At this branching point, the prevailing solution branch becomes unstable and a new branch is born. Solutions on this new branch have twice the period of the previous limit cycle. In the frequency domain, this corresponds to the occurrence of a subharmonic at half the previous frequency. The third possible loss of stability is through a Hopf bifurcation of the periodic solution (generalized Hopf bifurcation or torus bifurcation). This case is characterized by the crossing over the unit cycle of two complex conjugated eigenvalues [Fig. 3(c)] and corresponds to the onset of quasiperiodic oscillations.

\section{DYNAMIC ANALYSIS USING XPPAUT}

A wide collection of useful numerical algorithms for the exploration of ordinary differential equations has been made available through the public domain software XPPAUT [7]. With its graphical interface to the popular continuation and bifurcation software AUTO [8], XPPAUT combines the advantages of two worlds: A set of ordinary differential equation can be integrated with the phase plane explorer XPP until a steady-state has been reached; once balanced, the system equations can then be passed to AUTO for continuation and bifurcation analysis. A convenient text-based interface allows the differential equations to be entered without the need for tedious low-level programming in FORTRAN or C. Fig. 4 shows a screenshot of the main window of XPPAUT with the embedded AUTO in the foreground.

To arrive at an analytical model for the circuit in Fig. 1, the fluxcurrent linkage has been approximated by an $n$ th-order polynomial for the current $i_{2}$ as a function of the magnetic flux $\Phi$

$$
i_{2}=a_{1} \cdot \Phi+a_{n} \cdot \Phi^{n} .
$$

Using Kirchhoff's law, the following set of differential equation can be derived:

$$
\begin{aligned}
{\left[\begin{array}{c}
\dot{\Phi}_{1} \\
\dot{\Phi}_{2}
\end{array}\right] } & =\left[\begin{array}{cc}
0 & 1 \\
A_{3} & A_{1}
\end{array}\right] \cdot\left[\begin{array}{c}
\Phi_{1} \\
\Phi_{2}
\end{array}\right] \\
& +\left[\begin{array}{c}
0 \\
A_{0} V_{0} \cos \left(\omega_{0} t+\alpha\right)+A_{2} \Phi_{1}^{n-1} \Phi_{2}+A_{4} \Phi_{1}^{n}
\end{array}\right]
\end{aligned}
$$




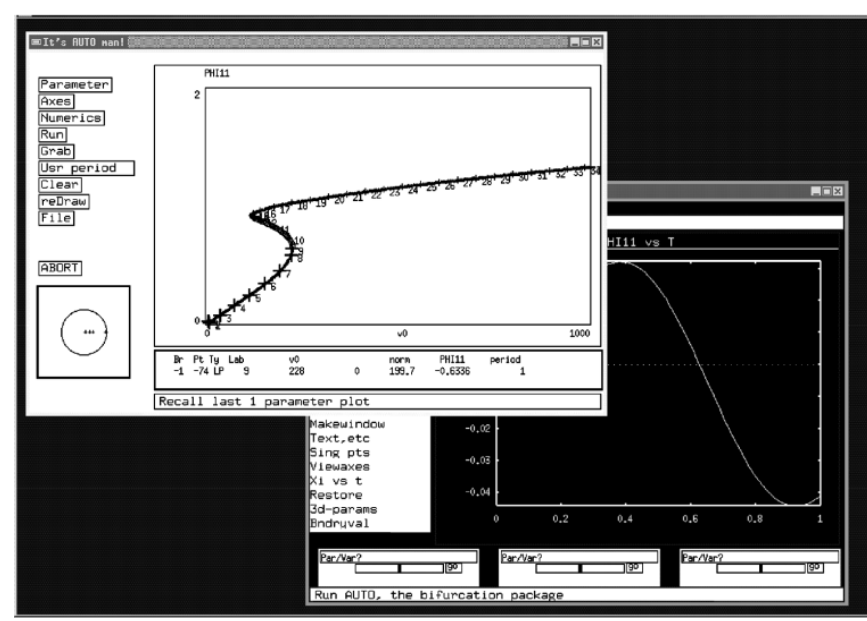

Fig. 4. Screenshot of the XPPAUT interface to AUTO.

where

$$
\begin{aligned}
& K_{\text {norm }}=\frac{1}{N}\left(1+\frac{R_{s}}{R_{p}}\right)^{-1} \\
& A_{0}=\omega \cdot K_{\text {norm }} \\
& A_{1}=-\left(R_{s} a_{1}+\frac{N}{R_{p} C}\right) \cdot K_{\text {norm }} \\
& A_{2}=-R_{s} a_{n} n \cdot K_{\text {norm }} \\
& A_{3}=-\frac{a_{1}}{C} \cdot K_{\text {norm }} \\
& A_{4}=-\frac{a_{n}}{C} \cdot K_{\text {norm. }}
\end{aligned}
$$

This nonautonomous system of nonlinear ordinary differential equations can now be turned into an equivalent autonomous system through the introduction of the dummy variable $\vartheta=\omega t$

$$
\left\{\begin{array}{l}
\frac{d \phi_{1}}{d t}=\phi_{2} \\
\frac{d \phi_{2}}{d t}=A_{3} \phi_{1}+A_{1} \phi_{2}+A_{0} V_{0} \cos (\vartheta)+A_{2} \phi_{1}^{n-1} \phi_{2}+A_{4} \phi_{1}^{n} \\
\frac{d \vartheta}{d t}=\omega_{0} .
\end{array}\right.
$$

Substituting the definition of the radian frequency $\omega_{0}=2 \pi / \mathrm{T}_{0}$ and rescaling the time variable as $\tau=\mathrm{t} / \mathrm{T}_{0}$ yields the following normalized system of equations. (Please see (5), shown at the bottom of the page.) Equation (5) forms the basis of the following analysis. Optionally, a set of boundary value conditions can be defined. These conditions can then be used to solve the system equations by considering the corresponding boundary value problem (BVP). Both XPP and AUTO have built-in BVP solvers based on powerful shooting algorithms [6]. To ensure $\mathrm{T}_{0}$-periodicity of the solutions, the following set of boundary conditions can be used (notice that the normalized time $\tau$ ranges from 0 to 1 when $t$ varies from 0 to $\mathrm{T}_{0}$ )

$$
\left\{\begin{array}{l}
\phi_{1}(0)=\phi_{1}(1) \\
\phi_{2}(0)=\phi_{2}(1) \\
\vartheta(0)=0
\end{array}\right.
$$

A slightly shortened version of the XPPAUT problem definition file used in the subsequent analysis of the circuit is given in the Appendix.

The problem definition file specifies a number of algorithmic options of XPP and AUTO. Noteworthy is the XPP option total $=1$ which fixes the final time of the integration algorithm at one period $\left(\tau=1\right.$ or $t=\mathrm{T}_{0}$ ). This parameter is read by AUTO to determine the period of the solutions to be continued. The option autovar $=$ phil tells AUTO to use $\phi_{1}$ on the ordinate of the bifurcation diagram. The corresponding range is chosen from 0 to $2.5 \times 10^{-3} \mathrm{~Wb}$.

Upon loading the above problem definition file into XPPAUT, an initial steady-state solution can be sought. The easiest way to achieve this is to use the boundary value problem solver of XPP; the relevant menus can be accessed using the mouse or via the key-stroke sequence $(\mathrm{B})$ ndryval $\rightarrow(\mathrm{S})$ how. To improve the numerical stability of the iterations, the variable $\vartheta$ can be reset to 0 every time it reaches or exceeds $2 \pi$. This cylindrical state space is defined using the sequence ph(A)sespace $\rightarrow$ (C)hoose, followed by ENTER to accept the default maximum value of $2 \pi$ and the choice of $\vartheta$ as the variable to be restricted.

Once a steady-state solution has been found, AUTO can be invoked through the key sequence $(\mathrm{F})$ ile $\rightarrow$ (A)uto. The AUTO window appears and should be made the active window. After having ensured that the ordinate shows the maximum value of the display variable $\phi_{1}$, the continuation procedure can be started: $(\mathrm{A})$ xes $\rightarrow(\mathrm{H}) \mathrm{i}$, followed by a click on the $\mathrm{OK}$ button, then $(\mathrm{R}) \mathrm{un} \rightarrow(\mathrm{P})$ eriodic. As the continuation proceeds, the small area in the bottom left-hand corner of the AUTO window displays the evolution of the eigenvalues of the monodromy matrix. Loss of stability through any of the above scenarios can thus be directly observed. Notice that one of the three eigenvalues remains fixed at +1 . This particularity is inherent to the continuation of periodic solutions. The observed eigenvalue corresponds to translations of the system states in the direction of the trajectory of the associated Poincare map. Subsequent states, which are found along this direction, exhibit a fixed distance of $\Delta \mathrm{t}=\mathrm{T}_{0}$; they are thus neither subject to compression (Floquet multiplier inside the unit circle) nor to expansion (Floquet multiplier outside the unit circle).

Upon completion of the continuation step, the trace of a solution branch can be inspected for bifurcations and branching points. The menu item $(\mathrm{G})$ rab allows navigation on a solution branch and reveals useful information of the established solutions. Various co-dimension-1 bifurcations such as limit points (fold bifurcation, denoted by LP), period doubling bifurcations (PD), and torus bifurcations (TR) can thus be identified with ease. Branching points at which the stability remains unchanged are labeled BP. Upon reaching a period doubling bifurcation (PD), the emerging branch can be continued using (R)un $\rightarrow$ (D)oubling. The direct visual feedback provided makes XPPAUT a powerful tool [e.g., convergence problems can easily

$$
\left\{\begin{array}{l}
\frac{d \phi_{1}}{d \tau}=T_{0} \phi_{2} \\
\frac{d \phi_{2}}{d \tau}=T_{0}\left(A_{3} \phi_{1}+A_{1} \phi_{2}+A_{0} V_{0} \cos (\vartheta)+A_{2} \phi_{1}^{n-1} \phi_{2}+A_{4} \phi_{1}^{n}\right) \\
\frac{d \vartheta}{d \tau}=2 \pi
\end{array}\right.
$$




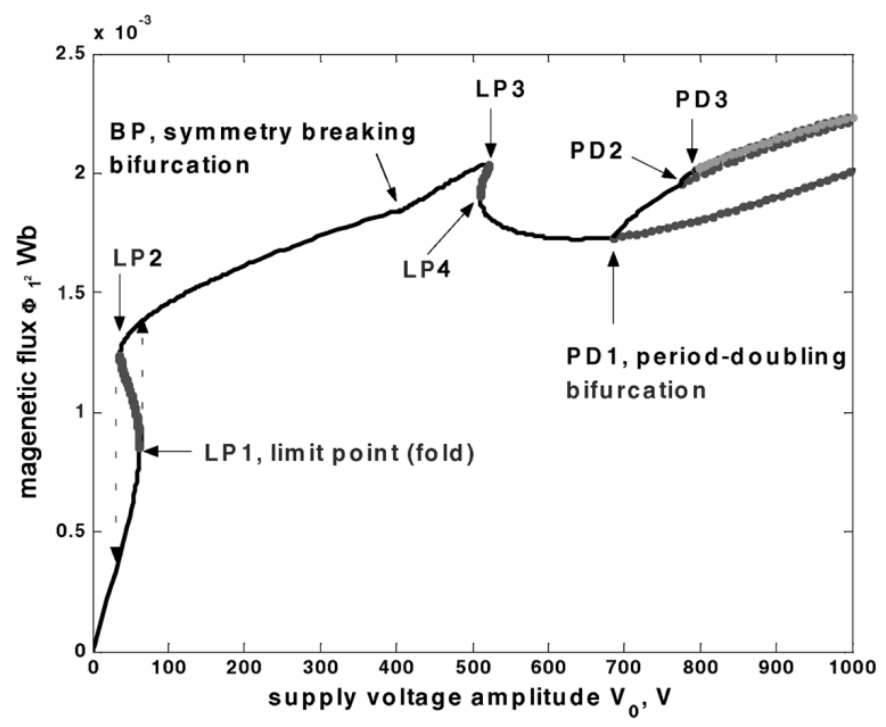

Fig. 5. Bifurcation diagram of the magnetic flux $\phi_{1}$ with parameter $V_{0}$.

be spotted (and remedied) as the continuation proceeds]. Fig. 5 shows the results of this interactive exploration of the solution space of the circuit. The bifurcation diagram of the magnetic flux $\left(\Phi_{1}\right)$ for varying supply voltage amplitudes $\left(V_{0}\right)$ is shown. This graph is the result of a number of continuation runs, tracing the path of the fundamental periodic solution $\left(\mathrm{T}_{0}=20 \mathrm{~ms}\right.$ or $\mathrm{f}_{0}=50 \mathrm{~Hz}$ ), as well as of two of the subharmonic oscillations encountered $\left(2 \mathrm{~T}_{0}\right.$ and $\left.4 \mathrm{~T}_{0}\right)$.

\section{DISCUSSION}

Variation in the supply voltage amplitude $\mathrm{V}_{0}$ can cause the flux to undergo a number of spontaneous transitions. In a voltage sweep from 0 to $1000 \mathrm{~V}$, the following observations can be made: Upon reaching the limit point LP1, the magnetic flux suddenly jumps from $0.8 \times 10^{-3} \mathrm{~Wb}$ to $1.4 \times 10^{-3} \mathrm{~Wb}$. Near $400 \mathrm{~V}$, a symmetry breaking bifurcation is observed, followed by a second jump phenomenon close to $500 \mathrm{~V}$ (LP3, cf. Fig. 5). The loss of symmetry can easily be spotted from a bifurcation diagram in which both the maximum and minimum of the periodic solutions are displayed. In AUTO, this can be achieved through the key sequence (A)xes $\rightarrow h(I)-$ lo. The result is shown in Fig. 6.

Fig. 7 is an enlargement of the cascade of period doubling bifurcations which can be found above $680 \mathrm{~V}$. Upon reaching a period doubling bifurcation, the prevailing T-periodic solution becomes unstable and is replaced by a solution with $\mathrm{T}^{\prime}=2 \mathrm{~T}$. The continuation of the emerging branch reveals further period doubling bifurcations (PD2, PD3,...). The corresponding phase portraits are of a progressively growing complexity (Fig. 8). Near $800 \mathrm{~V}$, the period has become infinite and a strange attractor is observed.

The period doubling route to chaos was first studied by $\mathrm{M}$. J. Feigenbaum, who found that the sequence of bifurcation parameters $\left\{\mathrm{a}_{k}\right\}$ obeys a geometric law with a universal constant [11]. The Feigenbaum constant has been defined as the limit

$$
\delta=\lim _{k \rightarrow \infty}\left\{\frac{a_{k}-a_{k-1}}{a_{k+1}-a_{k}}\right\} \approx 4.6692016 \ldots
$$

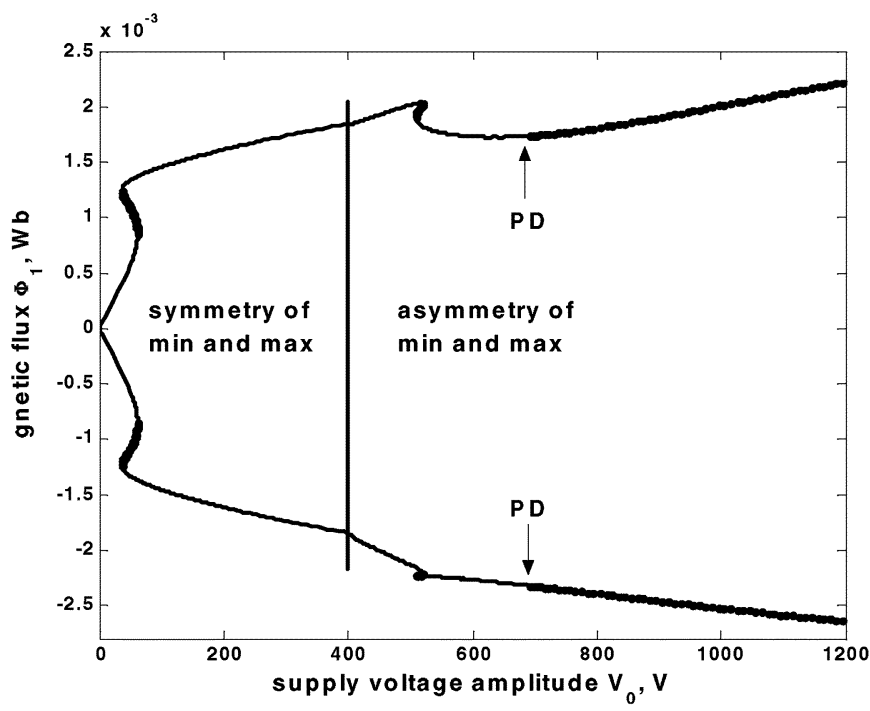

Fig. 6. Symmetry breaking bifurcation near $400 \mathrm{~V}$.

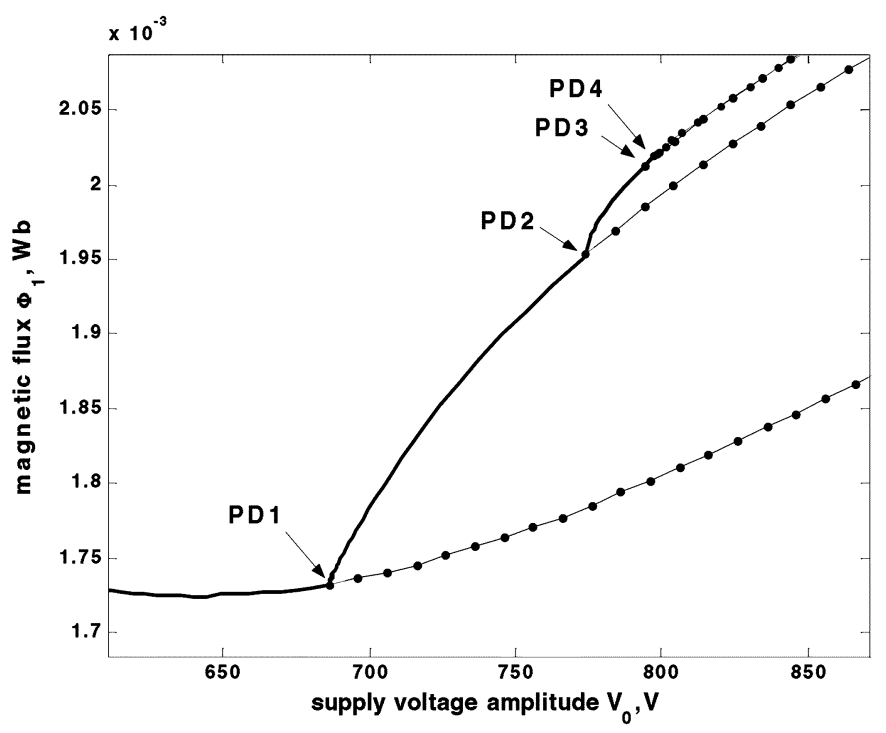

Fig. 7. Cascade of period doublings leading to a strange attractor near $800 \mathrm{~V}$.

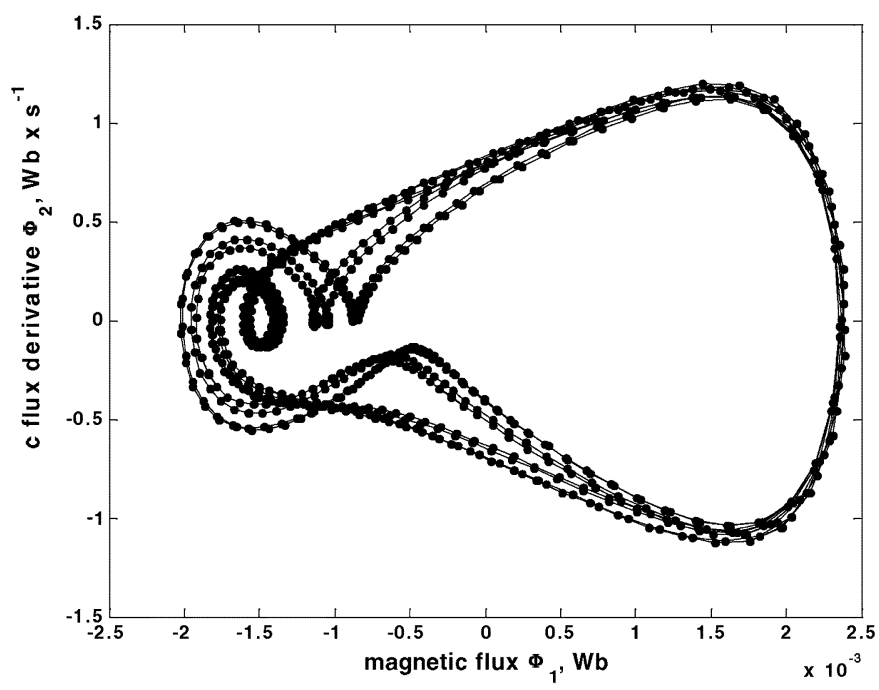

Fig. 8. Period-8 limit cycle near PD4 $\left(V_{0} \approx 799 \mathrm{~V}\right)$.

In practical applications, however, the limit $\mathrm{k} \rightarrow \infty$ cannot be taken. Nevertheless, an estimate of the Feigenbaum constant can 


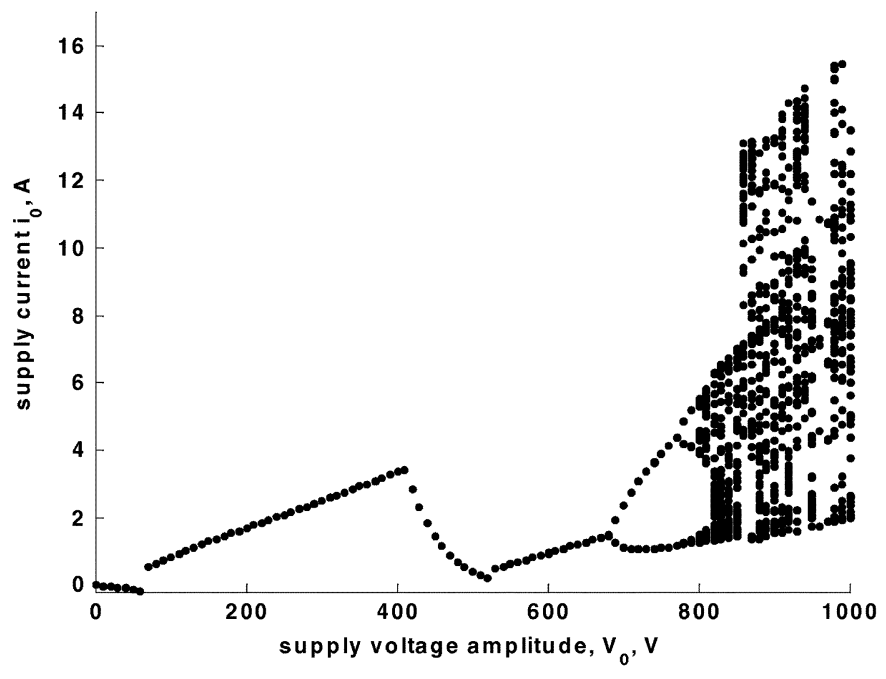

Fig. 9. Bifurcation diagram of the supply current $i$; onset of chaos near $800 \mathrm{~V}$.

be obtained from a finite sequence $\left\{\delta_{\mathrm{k}}\right\}$. The AUTO analysis yields the following sequence of bifurcation parameters:

$$
\begin{aligned}
& \text { PD1: } \mathrm{a}_{1}=686.3 \mathrm{~V} \\
& \text { PD2: } \mathrm{a}_{2}=774.2 \mathrm{~V} \\
& \text { PD3: } \mathrm{a}_{3}=794.8 \mathrm{~V} \\
& \text { PD4: } \mathrm{a}_{4}=799.3 \mathrm{~V} .
\end{aligned}
$$

Using these values, the following two estimates $\underline{\delta}_{1}$ and $\underline{\delta}_{2}$ are found:

$$
\begin{aligned}
& \underline{\delta}_{1}=\frac{774.2-686.3}{794.8-774.2} \approx 4.26699 \\
& \underline{\delta}_{2}=\frac{794.8-774.2}{799.3-794.8} \approx 4.57778 .
\end{aligned}
$$

These results are in good accordance with the Feigenbaum scenario, supporting the hypothesized onset of chaos near $800 \mathrm{~V}$.

Fig. 9 shows the bifurcation diagram of the corresponding supply current $i$. This graph has been obtained through the time-domain simulation of the system equations (fourth-order Runge-Kutta algorithm, voltage increments of $2 \mathrm{~V}$ at a rate of 6 steps $/ \mathrm{min}$ ). The resulting time series is filtered using a stroboscopic map with the frequency of the supply $(50 \mathrm{~Hz}$, $\mathrm{T}_{0}=20 \mathrm{~ms}$ ). Fifty equidistant samples are taken from the last fifty periods of the simulated current corresponding to every voltage step. The onset of chaos through a cascade of period doublings near $800 \mathrm{~V}$ is clearly visible.

\section{CONCLUSION}

An interactive bifurcation analysis has been presented using the differential equation analyzer XPPAUT. Summarizing the principal ideas of pseudo-arc-length continuation and the stability analysis of periodic solutions, a detailed description is given of the XPPAUT problem definition file. Suitable boundary conditions are introduced to facilitate the establishment of an initial steady-state solution. A step-by-step guide of an interactive bifurcation analysis is given and the results are discussed. The observed onset of chaos following a cascade of period doubling bifurcations is successfully attributed to the Feigenbaum scenario. With its seamless integration of boundary value solvers, integration algorithms, and the continuation and bifurcation software AUTO, XPPAUT is shown to be an excellent tool for the analysis of nonlinear dynamic systems.

\section{APPENDIX \\ XPPAUT PRoBlem DeFINITION FILE}

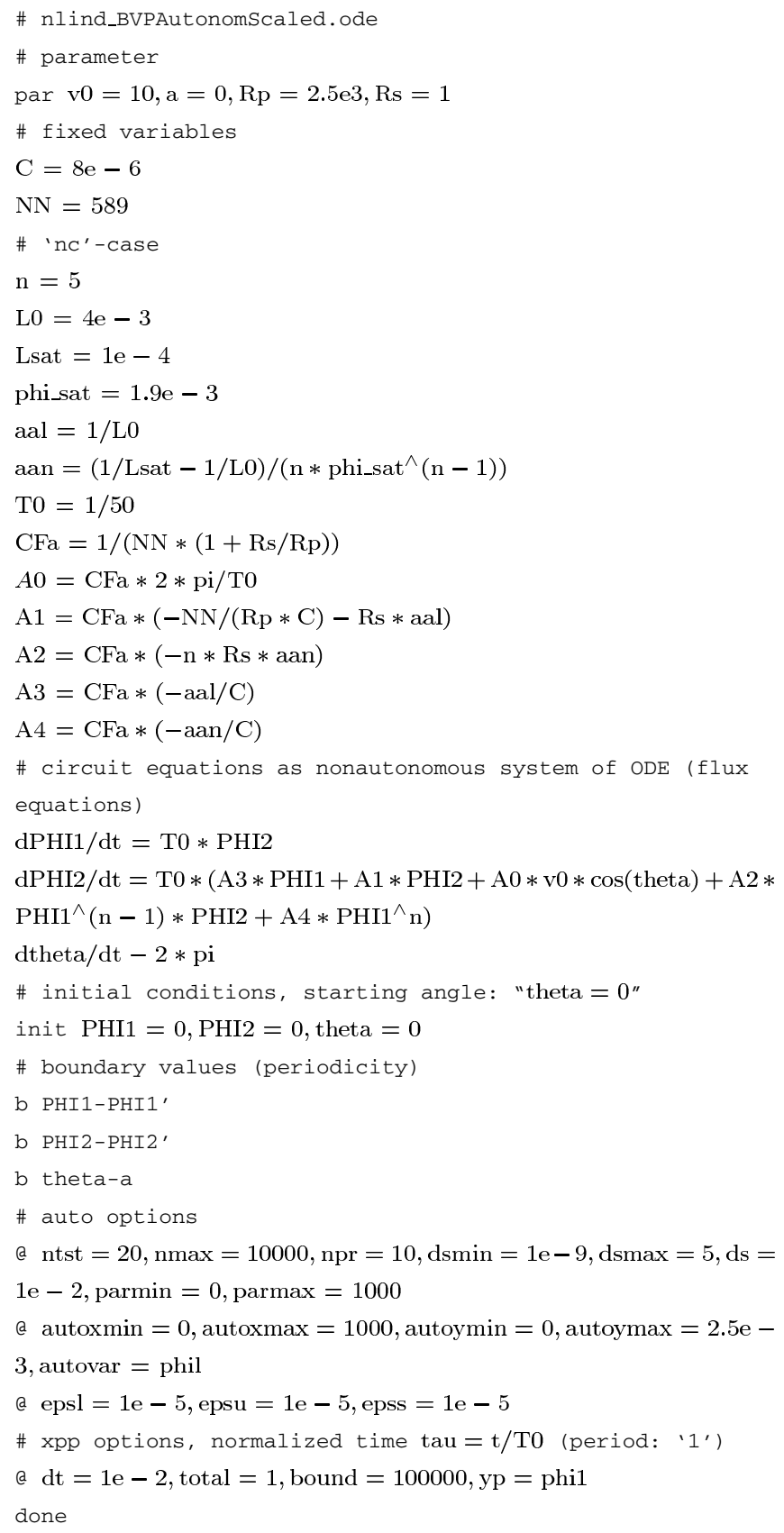

\section{REFERENCES}

[1] C. Kieny, "Application of the bifurcation theory in studying and understanding the global behavior of a ferroresonant electric power circuit," IEEE Trans. Power Del., vol. 6, no. 2, pp. 866-872, Apr. 1991.

[2] S. Mozaffari, S. Henschel, and A. C. Soudack, "Chaotic ferroresonance in power transformers," Proc. Inst. Elect. Eng., Gen. Transm. Distrib., vol. 142 , no. 3, pp. 247-250, 1995. 
[3] A. Ben-Tal, D. Shein, and S. Zissu, "Studying ferroresonance in actual power systems by bifurcation diagram,” Elect. Power Syst. Res., vol. 49, pp. 175-183, 1999.

[4] Z. Emin, B. A. T. Al-Zahawi, Y. K. Tong, and M. Ugur, "Quantification of the chaotic behavior of ferroresonant voltage transformer circuits," IEEE Trans. Circuits Syst. I, vol. 48, no. 6, pp. 757-760, Jun. 2001.

[5] T. S. Parker and L. O. Chua, "Chaos: A tutorial for engineers," Proc. IEEE, vol. 75, pp. 982-1008, 1987.

[6] B. Ermentrout, Simulating, Analyzing, and Animating Dynamical Systems-A Guide to XPPAUT for Researchers and Students, 1st ed. Philadelphia, PA: Soc. Industrial Appl. Math., 2002.

[7] — (2001) XPP-Aut-X-Windows Phase Plane Plus Auto [Online]. Available: http://www.math.pitt.edu/ bard/bardware/tut/start.html\#tech

[8] E. J. Doedel, R. C. Paffenroth, A. R. Champneys, T. F. Fairgrieve, Y. A. Kuznetsov, B. Sandstede, and X. J. Wang. (2001) AUTO 2000-Continuation and Bifurcation Software for Ordinary Differential Equations (With HomCont) [Online]. Available: ftp.cs.concordia.ca/pub/doedel/auto/auto.ps.gz.

[9] Y. A. Kuznetsov, Elements of Applied Bifurcation Theory, 2nd ed. New York: Springer Verlag, 1998.

[10] R. Seydel, Practical Bifurcation and Stability Analysis, 2nd ed. New York: Springer Verlag, 1994.

[11] J. Argyris, G. Faust, and M. Haase, An Exploration of Chaos, 1st ed. New York: North-Holland, 1994.

Frank Wörnle received degrees in telecommunications engineering from the Hochschule für Technik und Wirtschaft des Saarlandes, Saarbrücken, Germany, and the University of Metz, Metz, France, in 1994 and 1995, respectively. He received the Ph.D. degree from the School of Engineering, Science, and Design, Glasgow Caledonian University, Glasgow, U.K., in 2002.

He is now a lecturer in the School of Mechanical Engineering, University of Adelaide, Adelaide, Australia. His research interests lie in the area of artificial intelligence and robotic multiagent systems.
David K. Harrison received the M.Sc. and Ph.D. degrees in 1982 and 1986, respectively, from the University of Manchester, Manchester, U.K.

He was with Farranti Electronics Ltd as a production manager, with the Simon-Vicars Ltd as a CAD manager, and with the Staffordshire University, Stafford, U.K., as a Senior Lecturer before joining the Glasgow Caledonian University, Glasgow, U.K., as a Professor in 1994 . During his academic career, he published more than 300 papers and supervised more than 20 M.phil./Ph.D. students. His research interests lies in CAD, CAM, and non-linear phenomena in electromagnetic systems.

Chengke Zhou received the B.Sc. and M.Sc. degrees in 1983 and 1986, respectively, from the Electrical Department, the HuaZhong University of Science and Technology, Wuhan, China, and the Ph.D. degree in 1994 from the Electrical Engineering Department, Manchester University, Manchester, U.K..

He is now a lecturer with the School of Engineering, Science, and Design, the Glasgow Caledonian University, Glasgow, U.K. His research interests lie in the areas of electrical power systems, high-voltage engineering, and partial discharge detection for electrical machine condition monitoring. He has published more than 30 papers. 